\title{
Natural Bio-Polymer and Pharmaceutical Drugs in Textile Processing
}

\author{
Prof. (Dr.) Usha Sayed ${ }^{1}$, and Ravikant Sharma ${ }^{2}$
}

\begin{abstract}
Chitosan is a nontoxic, biodegradable, and biocompatible polysaccharide of $\mathrm{b}(1-4)$-linked D-glucosamine and $\mathrm{N}$ acetyl-D-glucosamine. This derivative of natural chitin presents remarkable properties that have paved the way for the introduction of chitosan in the biomedical and pharmaceutical fields. Chitosan is deacylated chitin. "Squid pens," waste shell by-products of squid processing, are a renewable and inexpensive source of Chitosan

In order to improve or impart new properties to chitosan, chemical modification of the chitosan chains, generally by either grafting of small molecules or polymer chains onto the chitosan backbone or by quarterisation of the amino groups, has been investigated. Chitosan chains possess three attractive reactive sites for chemical modification: two hydroxyl groups (primary or secondary) and one primary amine. The site of modification is dictated by the desired application of the final chitosan derivative.

Derivatives of chitin have been produced and used to impart antistatic and soil repellent characteristics to the textile. In textile industry, chitin can be used in printing and finishing preparations, while the Chitosan is able to remove dyes from dye processing effluents. Besides these, chitin and Chitosan both have made remarkable contribution to medical related textile sutures, threads and fibres. Chitosan is applied in the textile industry, as an antimicrobial finish, became popular due to its ability to provide protection against allergies and infection diseases, coupled with moisture retention and wound healing capabilities.

Tetracyclines are a group of broad-spectrum antibiotics whose general usefulness has been reduced with the onset of antibiotic resistance. The tetracyclines consist of a polycyclic ring with differing side chains and are a broad-spectrum class of antibiotics against aerobes and anaerobes. Tetracyclines are primarily bacteriostatic and exert their antimicrobial effect by the inhibition of protein synthesis. Tetracycline is active against a wide range of gram-negative and gram-positive organisms. The drugs in the Tetracycline class have closely similar antimicrobial spectra, and cross-resistance among them is common. Tetracycline is about $65 \%$ bound to plasma proteins (short-acting). The protein binding for intermediate and long-acting analogs is usually greater.

Tetracycline is a yellow, odorless, crystalline powder. Tetracycline is stable in air but exposure to strong sunlight causes it to darken. Its potency is affected in solutions of $\mathrm{pH}$ below 2 and is rapidly destroyed by alkali hydroxide solutions. Tetracycline is very slightly soluble in water, freely soluble in dilute acid and in alkali hydroxide solutions, sparingly soluble in alcohol, and practically insoluble in chloroform and in ether.
\end{abstract}

1and ${ }^{2}$ Department of Fibres and Textile Processing Technology, Institute of Chemical Technology, Matunga (E),Mumbai-400019, India.

The present research work deals with the functional value addition of the fabric, by a tangentially different approach namely antibiotics drugs available easily in the market has been used on various textile substrates like natural substrates and thus impart an antibacterial property to the treated fabrics.

This research work also deals with reuse of outdated antibiotics drugs in textile processing. Drugs lose their potency beyond their expiration date, and therefore their effectiveness and their ability to perform their function is affected. Antibiotics drugs which play an important role in treating several diseases become a colossal problem during disposal for various reasons. The common practice for discarding such outdated drugs is to dispose it in the landfill or in the garbage. Such methods still become irrelevant since it affects water supply and environment. Thus disposing of such drugs is a major problem in today's pharmaceutical Industries. The present study uses the discarded Drugs in textile processing.

This work depicts a successful attempt to provide antimicrobial finish to the silk fabric with chitosan and outdated tetracycline. This method of unused medicines valorisation can solve two major environmental and economic problems: limitation of environmental pollution with pharmaceutically active compounds and reduction of the disposal costs of expired drugs.

Keywords-Anti-biotics, Tetracycline hydrochloride, Expired drugs, chitosan, Silk

\section{INTRODUCTION}

Chitosan is a linear aminopolysaccharide of glucosamine and $\mathrm{N}$-acetylglucosamine units and is obtained by alkaline deacetylation of chitin extracted from the exoskeleton of crustaceans such as shrimps and crabs, as well from the cell walls of some fungi. Chitosan is a nontoxic, biodegradable, and biocompatible polysaccharide of b(1-4)-linked Dglucosamine and $\mathrm{N}$ acetyl-D-glucosamine. This derivative of natural chitin presents remarkable properties that have paved the way for the introduction of chitosan in the biomedical and pharmaceutical fields. In order to improve or impart new properties to chitosan, chemical modification of the chitosan chains, generally by either grafting of small molecules or polymer chains onto the chitosan backbone or by quarterisation of the amino groups, has been investigated. Chitosan chains possess three attractive reactive sites for chemical modification: two hydroxyl groups (primary or secondary) and one primary amine. The site of modification is dictated by the desired application of the final chitosan derivative [1].

Tetracyclines are a group of broad-spectrum antibiotics whose general usefulness has been reduced with the onset of antibiotic resistance. The tetracyclines consist of a polycyclic ring with differing side chains and are a broad-spectrum class of antibiotics against aerobes and anaerobes [2],[3]. Tetracyclines are primarily bacteriostatic and exert their antimicrobial effect by the inhibition of protein synthesis. Tetracycline is active against a wide range of gram-negative and gram-positive organisms. The drugs in the Tetracycline class have closely similar antimicrobial spectra, and cross- 
resistance among them is common [4]. Tetracycline is about $65 \%$ bound to plasma proteins (short-acting). The protein binding for intermediate and long-acting analogs is usually greater [5].

Tetracycline is a yellow, odorless, crystalline powder. Tetracycline is stable in air but exposure to strong sunlight causes it to darken. Its potency is affected in solutions of $\mathrm{pH}$ below 2 and is rapidly destroyed by alkali hydroxide solutions. Tetracycline is very slightly soluble in water, freely soluble in dilute acid and in alkali hydroxide solutions, sparingly soluble in alcohol, and practically insoluble in chloroform and in ether [6].

Degradation products of tetracycline accumulate within mitochondria of renal cells and interfere with oxidative phosphorylation and can potentially cause nausea, vomiting, lethargy, polydipsia, polyuria, glycosuria, aminoaciduria, phosphaturia, proteinuria, acidosis, and hypokalaemia [7][11]. Outdated tetracyclines should be thrown out. Some medications may instruct the user to discard in the toilet, if there is any leftover. This method may not be any safer than throwing in the trash, because it still is contaminating water supply [12]-[20].

Silk is an animal fibre and it is also called as protein fibre. Silk is an only fibre which is found in filament form. Silk is produced by insects. Fibroin is the main chemical component of silk [21].

Silk is a natural protein, like wool fibre, due to this, mechanism of dyeing silk is dependent not only on free amino and carboxyl groups but also on phenolic with accessible $\mathrm{OH}$ group. Because of slightly cationic character of silk with isoelectric point at above $\mathrm{pH} 5.0$, it can be dyed with anionic dye such as acid, metal complexes, reactive and selected direct dyes [22].

This work depicts a successful attempt to dye and provide antimicrobial finish of Silk fabric with chitosan and outdated tetracycline. This method of unused medicines valorisation can solve two major environmental and economic problems: limitation of environmental pollution with pharmaceutically active compounds and reduction of the disposal costs of expired drugs.

\section{DETAILS EXPERIMENTAL}

\subsection{Materials and Procedures}

- Commercially available ready for dye (RFD) Silk fabric was used for the study.

- Tetracycline Hydrochloride (TCH) by Piramal Healthcare Limited, Gujrat, (Batch No. 1558, Mfd. Jun 2010, Exp.-May 2012)

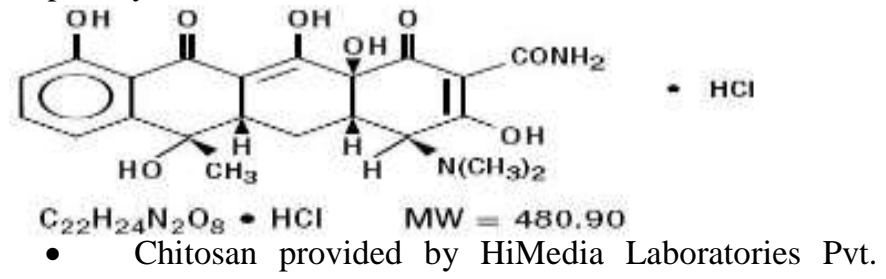
Ltd., Acetic Acid

2.2 Procedure for Preparation of chitosan derivatives in presence of TCH (Antibiotic Drug)
Reaction was carried out at atmospheric pressure in a three neck flask. The mechanical stirrer was used and the speed of the stirrer was maintained by using knob. The thermometer was fixed to the side neck of the flask through the thermometer pocket. The assembly was heated using heating mantel. Vacuum grease was applied on the joints of three necks to make it air tight.

The chemicals namely Chitosan, Acetic Acid, Water was taken as a reactant in three necked flask and stirred for 2 hours at $60^{\circ} \mathrm{C}$ under reflux condition after which TCH was added slowly and the reaction was carried out for $2 \mathrm{hrs}$. The FTIR obtained for the mixture and the compared the FTIR of chitosan is different and is as seen below in the figure- 2 .

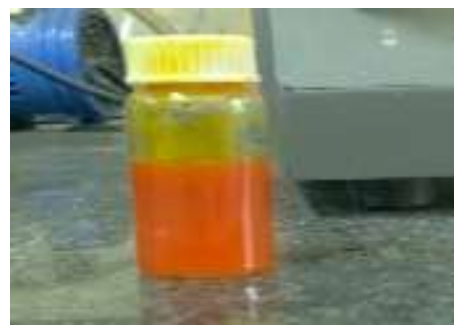

Fig. 1 Sample-A

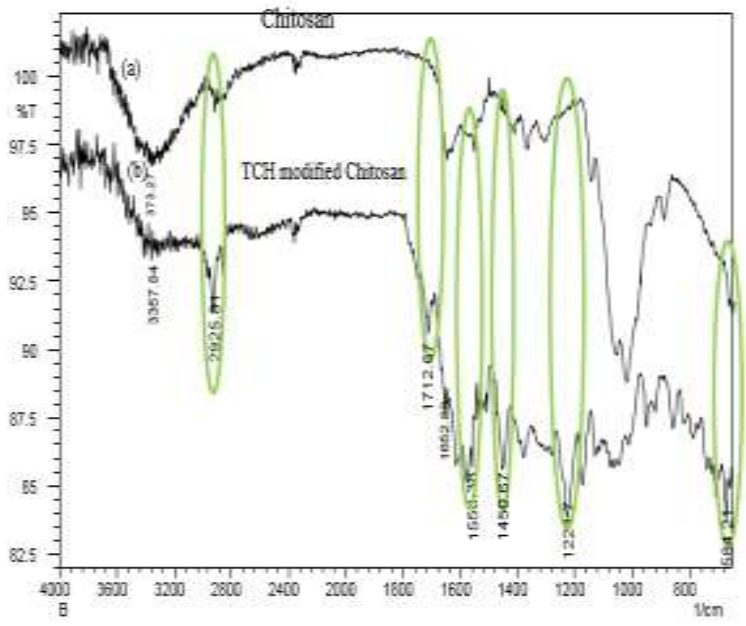

Fig. 2: FTIR of (a) Chitosan; (b) derivative of chitosan using TCH

2.3 Dyeing and antimicrobial finishing of Silk by the above product

$1 \%$ stock solution of above chitosan derivatives was prepared for dyeing. Silk was dyed with above prepared chitosan-TCH derivative, keeping liquor ratio of $15: 1$, at $80^{\circ} \mathrm{C}$ for $40 \mathrm{~min}$ in Rota dyer machine. Then rinsed and dried.

Dyed samples were evaluated for the depth of the colour by determining K/S values using a Spectraflash $\square$ SF 300, Computer Colour Matching System supplied by Data colour International, U.S.A. An average of four readings taken at four different sample areas, was used to calculate the reflectance values.

\section{RESUlTS AND DISCUSSIONS}

Silk is a protein fibre containing different types of amino acids and is well known for its brilliancy.Silk is being lately 
used for fashion apparels and has its own typical look.

In this project an attempt has been made to finish the fabric with a value added finish namely antimicrobial finish to increase its shelf-life and also the areas of application of silk fibres and fabrics.

Chitosan, a natural biopolymer is well known for imparting anti-microbial properties was used to finish silk fabric, by the exhaust method. Anti-microbial properties were obtained and is as seen in Table I.

The zone formed, $23 \mathrm{~mm}$ in diameter for E. coli (ATCC 43894 ) and $16 \mathrm{~mm}$ in diameter for S. aureus (ATCC 29213) after $18 \mathrm{hrs}$ of incubation showed the antimicrobial effect of the treated Silk fabric (Fig-3)

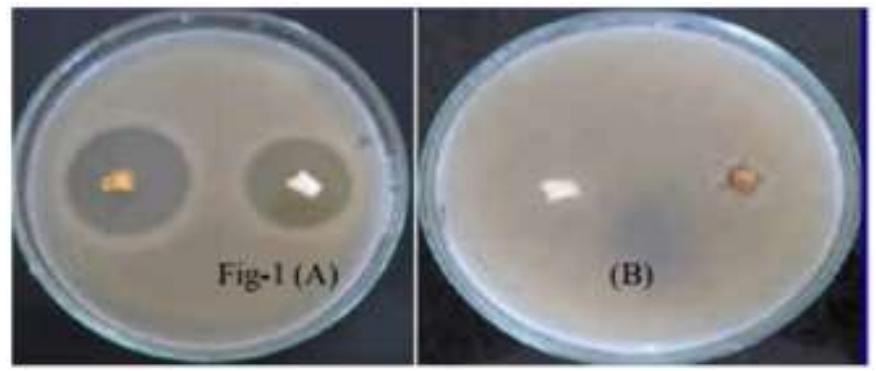

Fig-3 (A) Zone of inhibition against E. coli (ATCC 43894) and S. aureus (ATCC 29213) exhibited by synergistic drug treated Silk. (B) Zone of inhibition against E. Coli (ATCC 43894) and S. aureus (ATCC 29213) exhibited by untreated Silk.

TABLE I. ANTI-MICROBIAL ACTIVITY OF SILK FABRICS

\begin{tabular}{|c|c|c|}
\hline Types of micro-organism & Substrate & Inhibition Zone (mm) \\
\hline \multirow{3}{*}{ E. coli } & Untreated Silk & 0 \\
\cline { 2 - 3 } & Silk treated with Chitosan & 9 \\
\cline { 2 - 3 } & Silk treated with TCH & 22 \\
\cline { 2 - 3 } & Silk treated with Chitosan and TCH & 23 \\
\hline \multirow{4}{*}{ S. aureus } & Untreated Silk & 7 \\
\cline { 2 - 3 } & Silk treated with Chitosan & 15 \\
\cline { 2 - 3 } & Silk treated with TCH & 16 \\
\cline { 2 - 3 } & Silk treated with Chitosan and TCH & 0 \\
\hline
\end{tabular}

On further dyeing the silk fabric with $\mathrm{TCH}$ drug both colour value and anti-microbial values are obtained as seen in (Table I and II). The above result encouraged the prepare product mixture chitosan and $\mathrm{TCH}$ as given above and apply the same on the silk fabric by using rota dyer machine at liquor ratio of $15: 1$, at $80^{\circ} \mathrm{C}$ for $40 \mathrm{~min}$. On comparing the antimicrobial properties imparted to the fabric as in (Table I), $\mathrm{TCH}$ mixture gives maximum antimicrobial value, followed by chitosan but surprisingly the product mixture gives the maximum anti-microbial value as given in the Table I. Chitosan does not impart colour value to the fabric as it is colorless but gives good antimicrobial values and tensile strength.

TCH drug on application on the silk fabric, imparts color value and good antimicrobial values as seen in (Tables I and II) When the product mixture of chitosan and $\mathrm{TCH}$ was used to finish the silk fabric, it imparts dark yellow colour to the fabric as seen in (Table II) more than silk fabric dyed with $\mathrm{TCH}$ alone.

TABLE II. COLOR STRENGTH VALUES OF DYED (TCH) SILK FABRICS

\begin{tabular}{|l|l|l|l|l|l|l|l|l|}
\hline $\begin{array}{l}\text { Sr. } \\
\text { No. }\end{array}$ & Sample & L* & $\mathbf{a}^{*}$ & $\mathbf{b}^{*}$ & $\mathbf{C}^{*}$ & $\mathbf{H}^{*}$ & $\begin{array}{l}\text { \% Colour } \\
\text { Strength }\end{array}$ & K/S \\
\hline 1. & Untreated silk fabric & 78.01 & 0.461 & 10.86 & 10.88 & 87.54 & 134.505 & 0.5353 \\
\hline 2 & TCH and silk fabric & 85.994 & -1.374 & 14.711 & 14.714 & 95.372 & 480.162 & 1.7221 \\
\hline 3 & Product and silk fabric & 87.149 & -1.024 & 15.845 & 15.874 & 93.74 & 587.21 & 2.411 \\
\hline
\end{tabular}

TABLE III FASTNESS PROPERTIES OF DYED (TCH) SILK FABRICS

\begin{tabular}{|c|c|c|c|c|c|c|c|}
\hline \multirow[t]{2}{*}{ Sr.No. } & \multirow{2}{*}{ Silk dyed with } & \multirow{2}{*}{$\begin{array}{l}\text { Washing } \\
\text { Fastness }\end{array}$} & \multirow{2}{*}{$\begin{array}{l}\text { Light } \\
\text { Fastness }\end{array}$} & \multicolumn{2}{|c|}{ Rubbing Fastness } & \multicolumn{2}{|c|}{ Fastness to perspiration } \\
\hline & & & & Dry & Wet & Alkaline & Acidic \\
\hline 1 & $\mathrm{TCH}$ & $3-4$ & $6-7$ & $4-5$ & $3-4$ & $3-4$ & $3-4$ \\
\hline 2 & Product & $4-5$ & $7-8$ & 5 & $4-5$ & $4-5$ & $4-5$ \\
\hline
\end{tabular}


TABLE IV CREASE RECOVERY AND STRENGTH PROPERTIES OF SILK FABRICS

\begin{tabular}{|l|l|l|l|l|}
\hline \multirow{2}{*}{ Substrate } & \multirow{2}{*}{ Curing Temp \& time } & \multirow{2}{*}{$\begin{array}{l}\text { Total } \\
(\mathrm{W}+\mathrm{F}), \text { deg. }\end{array}$} & & \multicolumn{2}{l|}{ Tensile Strength(kgf) } \\
\cline { 3 - 5 } & & 205 & Warp Way & Weft Way \\
\hline Untreated Silk & - & 210 & 16.40 & 14.65 \\
\hline \multirow{2}{*}{ Silk Dyed with TCH } & $170^{0} \mathrm{C} \& 3 \mathrm{mins}$ & 209 & 19.48 & 14.58 \\
\cline { 2 - 5 } & $170^{\circ} \mathrm{C} \& 4 \mathrm{mins}$ & 210 & 18.80 & 16.86 \\
\hline \multirow{2}{*}{ Silk Dyed with Product } & $170^{0} \mathrm{C} \& 3 \mathrm{mins}$ & 211 & 20.94 & 17.85 \\
\cline { 2 - 5 } & $170^{0} \mathrm{C} \& 4 \mathrm{mins}$ & 20.16 & 17.26 \\
\hline
\end{tabular}

Silk fabric treated with the product mixture of chitosan and $\mathrm{TCH}$ also gives the good fastness properties as compared to silk fabric treated with TCH as seen in Table III. Tensile strength and crease recovery angle properties of the silk fabric treated with the mixture of chitosan and TCH increased as compared to the silk fabric treated with only $\mathrm{TCH}$ seen in Table IV The above results are very encouraging, and a synergism between chitosan and TCH drug is observed giving increased antimicrobial activity. It opens another venue for recycling of drugs and its use in textile processing and hence a good method of disposal of the drugs too.

\section{CONCLUSION}

The above project is an innovative method of using the natural bio-polymer for reuse of expired drugs in textile processing. Here the product mixture of chitosan and $\mathrm{TCH}$ gave dyeing of silk imparting antimicrobial property which is a great value addition for apparel. The above experimentation has exhibited uniform level dyeing of silk can be achieved by using chitosan and expired TCH.

\section{ACKNOWLEDGEMENT}

The Authors are thankful to the Department of Fiber and Textile Processing Technology, Institute of Chemical Technology, Matunga, Mumbai for providing infrastructural facilities and Department of Science and Technology (DST) India for providing finanical support for this research work.

\section{REFERENCES}

[1] Raphae"1 Riva, He'loı"se Ragelle, Anne des Rieux, Nicolas Duhem ,(2011) Chitosan and Chitosan Derivatives in Drug Delivery and Tissue Engineering, Adv Polym Sci (2011) 244: 19-44 (Springer-Verlag Berlin Heidelberg) https://doi.org/10.1007/12_2011_137

[2] https://en.wikipedia.org/wiki/Tetracycline_antibiotics

[3] http://cellgro.com/media/upload/file/productinfosheets/new/Tetracyclin e\%20Hydrochloride.pdf

[4] http://www.drugs.com/pro/tetracycline.html

[5] http://www.drugs.com/ppa/tetracycline-hydrochloride.html

[6] Andrés R. Sánchez, Roy S. Rogers III, and Phillip J. Sheridan,Tetracycline and other tetracycline-derivative staining of the teeth and oral cavity. Int J Dermatol. 2004 Oct;43(10):709-15. https://doi.org/10.1111/j.1365-4632.2004.02108.x

[7] http://www.accessdata.fda.gov/drugsatfda_docs/label/2015/050278s037 lbl

[8] Prashant Gangawane \& Usha Sayed (2013), Innovative Use of Outdated Tetracycline Hydrochloride Drug to Dye Jute International Journal of Medicine and Pharmaceutical Sciences (IJMPS) Vol. 3, Issue 4, 23-28

[9] http://www.lindagreenwall.co.uk/docs/adt_nov_newsomegreenwall_v3.pdf (accessed on July 18, 2013).
[10] Daniel C. N. Chan, Gregory Shayne Rozier, Angela Steen, William D. Browning, Mahmood S. Mozaffari, Standardized method to produce tetracycline-stained human molar teeth in vitro, Quintessence Int ,2006;37:637-646

[11] Gross JM. Fanconi Syndrome (adult type)developing secondary to the ingestion of outdated tetracycline. Ann Intern Med 1963;58:523-8. https://doi.org/10.7326/0003-4819-58-3-523

[12] Frimpter GW, Timpanelli AE, Eisenmenger WJ, etal. Reversible "Fanconi Syndrome" caused by degraded tetracycline. JAMA 1963:184;111-3. https://doi.org/10.1001/jama.1963.03700150065010

[13] Montoliu J, Carrera M, Darnell A, Revert L. Lactic acidosis and Fanconi's syndrome due to degraded tetracycline. Br Med J (Clin Res Ed) $1981 ; 283: 1576-7$ https://doi.org/10.1136/bmj.283.6306.1576-a

[14] Fulop M, Drakin A. Potassium-depletion syndrome secondary to nephropathy apparently caused by "outdated tetracycline." New Engl J Med 1965;272:986-9. https://doi.org/10.1056/NEJM196505132721902

[15] Wegienka LC, Weller JM. Renal tubular acidosis caused by degraded tetracycline. Arch Intern Med 1964;114:232-5. https://doi.org/10.1001/archinte.1964.03860080082007

[16] Hemstreet BA. Antimicrobial-associated renal tubular acidosis. Ann Pharmacother 2004;38:1031-8. https://doi.org/10.1345/aph.1D573

[17] http://www.surgeryencyclopedia.com/St-Wr/Tetracyclines.html (accessed on July 18, 2013).

[18] Prashant Gangawane, Dr. Usha Sayed (2013), Dyeing of silk with discarded tetracycline hydrochloride drug, Asian J. Pharm. Tech., 3(1), 34-36

[19] Prashant Gangawane, Usha Sayed (2013), Dyeing of Nylon with discarded tetracycline hydrochloride drug, International Journal of Textile and Fashion Technology, 3(1), 49-54

[20] Prashant Gangawane, Dr. Usha Sayed (2013), Dyeing of Wool with discarded tetracycline hydrochloride drug, Research Journal of Pharmacy and Technology, 6(3), 244-246

[21] http://textilefashionstudy.com/silk-fiber-physical-and-chemicalproperties-of-silk/

[22] Md. Koushic Uddin, Ms. Sonia Hossain (2010), A Comparative Study on Silk Dyeing with Acid Dye and Reactive Dye, IJET-IJENS Vol:10 No:06 